\title{
Transillumination optical tomography of tissue-engineered blood vessels: a Monte Carlo simulation
}

\author{
Gang Yao and Mark A. Haidekker
}

\begin{abstract}
A Monte Carlo technique has been developed to simulate the transillumination laser computed tomography of tissue-engineered blood vessels. The blood vessel was modeled as a single cylinder layer mounted on a tubular mandrel. Sequences of images were acquired while rotating the mandrel. The tomographic image was reconstructed by applying a standard Radon transform. Angular discrimination was applied to simulate a spatial filter, which was used to reject multiply scattered photons. The simulation results indicated that the scattering effect can be overcome with angular discrimination because of the thin tissue thickness. However, any refractive-index mismatch among the tissue, the surrounding media, and the mandrel could produce significant distortions in the reconstructed image. (C) 2005 Optical Society of America
\end{abstract}

OCIS codes: $110.6960,170.0110,120.7000,290.0290$.

\section{Introduction}

One of the goals of tissue engineering is to create a small-diameter blood vessel substitute that could be used for heart bypass surgery, lower extremity bypasses, and tissue transfer. ${ }^{1}$ Significant progress has been made in recent years toward engineering blood vessels from a patient's own cells ${ }^{2}$ so that the body's immune system will not reject the tissue. The ideal vessel substitute should resemble the native tissues in every respect and should have sufficient mechanical strength. ${ }^{3}$ However, the challenge remains to develop a noninvasive technique that can be used to examine the vessel's quality effectively. Besides the mechanical properties, it is also important to measure the uniformity and structural defects of the vessel substitute. A fast imaging tool that can be used to monitor real-time vessel growth would be highly desirable.

Optical imaging of biological tissues has been an active area of research in recent years ${ }^{4,5}$ because it is noninvasive and has good soft-tissue contrast. Near-

The authors are with the Department of Biological Engineering, University of Missouri-Columbia, 1406 E. Rollins Street, Columbia, Missouri 65211. G. Yao's e-mail address is yaog@missouri.edu.

Received 31 August 2004; revised manuscript received 19 January 2005; accepted 25 February 2005.

0003-6935/05/204265-07\$15.00/0

(C) 2005 Optical Society of America infrared light is usually used to enhance the imaging penetration depth. The major obstacle in optical imaging is that the tissue is highly scattering in the visible-to-near-infrared wavelength region. A number of optical imaging techniques have been proposed to reject multiply scattered photons. ${ }^{6-8}$ The severity of multiple scattering also depends on the tissue thickness. For thin tissue samples, such as the engineered vessel substitute (usually a couple of hundreds of microns), the multiply scattered photons can be readily controlled.

A Monte Carlo simulation technique was developed to study the possibility of using transillumination laser computed tomography (laser CT) to image an engineered tissue graft ${ }^{9}$ in an bioreactor environment. Compared with other promising optical imaging techniques, such as optical coherence tomography, ${ }^{10}$ laser CT is relatively simple to construct and can image a large sample area with an array detector. It is especially suitable for imaging of thin tissues, where multiple scattering is not severe. The capability of this technology for imaging sample geometrical uniformity was simulated. The effects of tissue scattering and refractive-index mismatch on the reconstructed images were studied in the simulation.

\section{Method}

The simulation setup is illustrated in Fig. 1. The tissue graft was wrapped around a cylindrical man- 


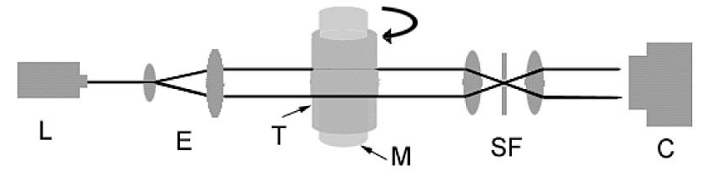

Fig. 1. Schematic setup of the optical tomography system: L, laser; E, beam expander; T, vessel; M, mandrel; SF, spatial filter; $\mathrm{C}$, array detector.

drel that was mounted inside a transparent container filled with index-matching fluid. The purpose of the index-matching solution was to reduce the refractiveindex mismatch of the surrounding medium and the tissue sample. ${ }^{11,12}$ The laser light was expanded by a beam expander to illuminate the whole section of the tissue sample. The transmitted light passed through a spatial filter and was detected by a CCD array detector. The spatial filter rejected those transmitted photons that had large deviation angles.

To construct a tomographic image of the tissue graft, we rotated the mandrel around its axis. At each rotation angle, the laser projection on the CCD was acquired. One sample cross section [two dimensional (2D)] was projected to a line of pixels [one dimensional (1D)]. By using the traditional backprojection algorithm, we reconstructed the $2 \mathrm{D}$ tomographic images from the $1 \mathrm{D}$ projection data, whereas threedimensional (3D) images can be reconstructed from the $2 \mathrm{D}$ projection data.

The Monte Carlo simulation has been used extensively in laser-tissue interaction studies. Usually the tissue is modeled as a multilayer slab structure. However, for our application, the tissue sample was modeled as a multilayer cylindrical structure (see Fig. 2). Each layer had a set of optical properties: refractive index $n$, absorption coefficient $\mu_{a}$, scattering coefficient $\mu_{s}$, and scattering anisotropy $g$. The surrounding medium was a transparent index-matching medium $\left(n_{0}, \mu_{a}=0, \mu_{s}=0\right)$. Two different types of mandrel materials were simulated (Fig. 2). The first configuration was an opaque solid mandrel [Fig. $2(\mathrm{a})$ ], and the second was a thin transparent tube [Fig. 2(b)].

A coordinate system was defined in the simulation (Fig. 2) in which the $x$ axis was aligned with the incident light direction and the $z$ axis was aligned with the axis of the cylinder layer. The origin of the coordinate system was located at the symmetric center of the tubular mandrel. The detector was located

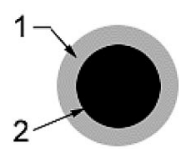

(a)
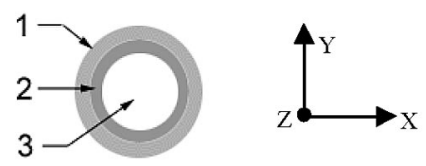

(b)
Fig. 2. Sample cross-sectional structure used in the Monte Carlo simulation. (a) Opaque mandrel: 1, tissue; 2, mandrel. (b) Translucent mandrel: 1 , tissue; 2 , mandrel; 3 , inner space of the mandrel. in the $y-z$ plane. The basic Monte Carlo simulation process was previously reported in detail. ${ }^{13}$ In the simulation, a photon packet was incident on the tissue graft through an index-matching medium. The photons were scattered and absorbed within the tissue. The movement of the photon packet was traced by using sampling theory according to the local scattering and absorption coefficients. The HeyneyGreenstein phase function ${ }^{14}$ was used to sample the scattering angle. At the interfaces of two layers with different refractive indices, the light was reflected or transmitted according to the Fresnel principle.

The tissue graft and the mandrel were modeled as cylinders with infinite height. A plane laser light was incident on the cylinder. The refractive index of the tissue graft was 1.37. The CCD chip has a pixel size of $24 \mu \mathrm{m}$ and dimensions of $256 \times 256$ pixels. A simple algorithm was applied to simulate the function of the spatial filter in which photons with exiting angles larger than a preset value were not scored. Within regions of nonscattering media, such as the transparent mandrel, direct ray tracing was used to track the photon movement.

In a laser CT system only the nonscattered (ballistic) photons can be used in image reconstruction because their trajectories are well defined. In the case of ballistic approximation, i.e., all photons propagate in a straight line, the projected light intensity on a CCD pixel $i$ can be calculated from a modified Beer's law:

$$
I^{i}=I_{0}^{i} \exp \left[-\int \mu_{t}(s) \mathrm{d} s\right],
$$

where $I_{0}{ }^{i}$ is the incident intensity for pixel $i, s$ is the path length, $\mu_{t}(s)$ is the attenuation coefficient along path $s$ and is the summation of the optical scattering coefficient $\mu_{s}$ and the optical absorption coefficient $\mu_{a}$ :

$$
\mu_{t}=\mu_{s}+\mu_{a} \text {. }
$$

Equation (1) can be rewritten in terms of sample attenuation $A$ :

$$
A^{i}=\ln \left(I_{0}^{i} / I^{i}\right)=\int \mu_{t}(s) \mathrm{d} s .
$$

The image of $\mu_{t}(s)$ can be reconstructed by using the standard Radon transform ${ }^{15}$ from the attenuation projections, which were calculated by the Monte Carlo simulation. And the filtered backprojection was actually used in the simulation to reconstruct the sample of the $\mu_{t}$ image. Because of the rotational symmetry, 100 projection scans were calculated in the simulation at a step size of $1.8^{\circ}$.

All the simulations were performed on a cluster of eight Linux workstations powered by Athlon $1.8 \mathrm{GHz}$ processors. For each projection, 1-8 $\times 10^{8}$ photon packets were used. Each projection took 34 min per $10^{8}$ photons on a single computer for samples with a 


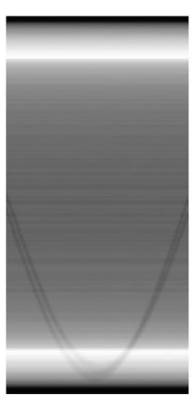

(a)

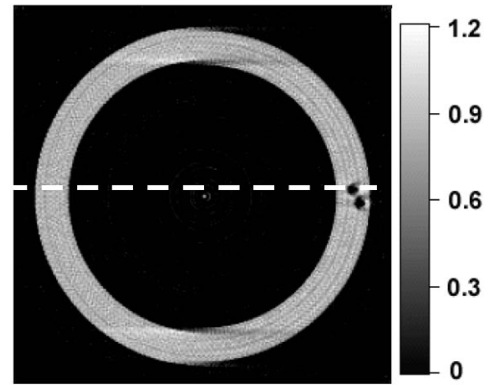

(b)

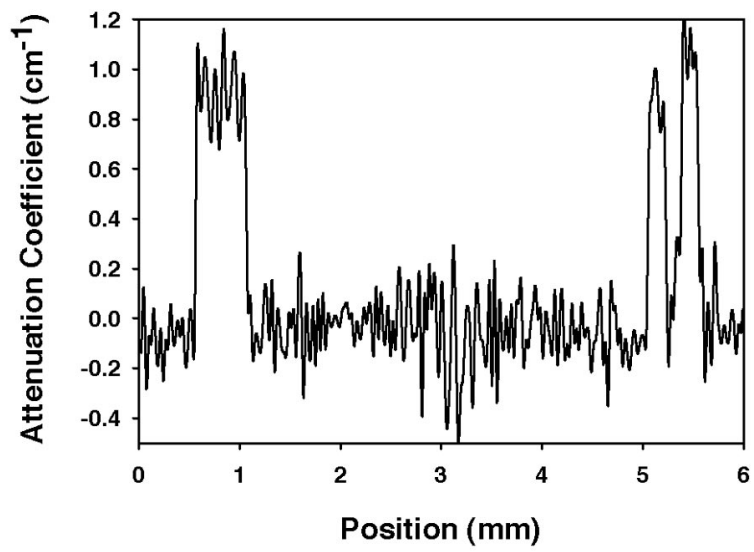

(c)

Fig. 3. Simulation results of the tissue cylinder. (a) Projection sinogram, (b) reconstructed tissue. The gray-scale bar indicates the value of the reconstructed attenuation coefficient $\mu_{t}$ (in inverted centimeters). (c) A line [marked in (b)] crossing one bubble object in (b).

scattering coefficient of $40 \mathrm{~cm}^{-1}$. Less time was needed for samples with smaller scattering coefficients.

\section{Results and Discussion}

To validate the simulation program, we simulated a sample with low scattering and no index mismatch with the surrounding medium. The optical properties of the tissue were $n=1.37, \mu_{a}=$ $0.2 \mathrm{~cm}^{-1}, \mu_{s}=1 \mathrm{~cm}^{-1}$, and $g=0.9$. The outer diameter of the tissue was $0.5 \mathrm{~cm}$, and the inner diameter of the tissue (or the mandrel diameter) was $0.4 \mathrm{~cm}$. There were two small sphere-shaped void regions buried inside the tissue. The void region was $80 \mu \mathrm{m}$ in radius and was filled with the same medium surrounding the sample.

Figure 3(a) shows the projection sinogram, which consisted of a series of projected attenuation profiles at angles from $0^{\circ}$ to $180^{\circ}$. The reconstructed image is shown in gray scale in which white indicates high attenuation and a dark color indicates low attenuation. It can be seen from Fig. 3(b) that the two void regions were imaged clearly and that their sizes and locations were reconstructed correctly. The reconstructed object has a diameter of $168 \mu \mathrm{m}$ and $x$ and $y$ coordinates (defined in Fig. 2) of 2256 and $96 \mu \mathrm{m}$, respectively, relative to the sample center, whereas

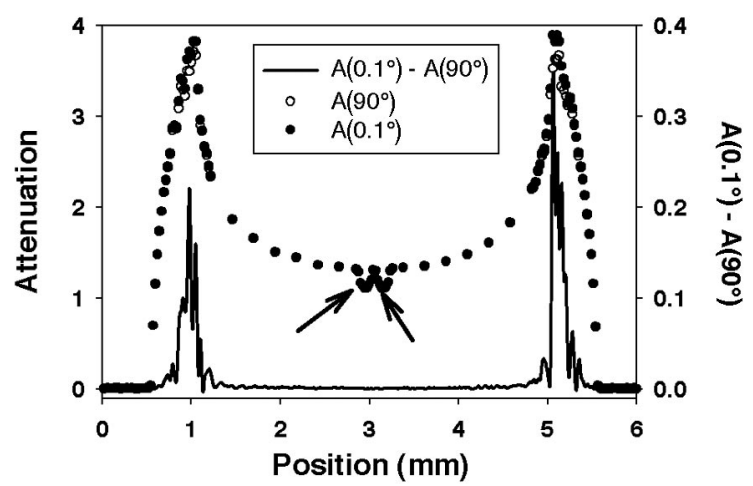

Fig. 4. Projection profile of a tissue sample with a scattering coefficient of $30 \mathrm{~cm}^{-1}: \mathrm{A}\left(90^{\circ}\right)$ is the attenuation measured without spatial filter and $\mathrm{A}\left(0.1^{\circ}\right)$ is the attenuation measured with a spatial filter that has an acceptance angle of $0.1^{\circ}$. The two arrows indicate the locations of the bubbles.

the actual diameter is $160 \mu \mathrm{m}$ and the actual coordinates are 2250 and $100 \mu \mathrm{m}$, respectively. In addition, Fig. 3(c) shows the reconstructed attenuation coefficient of the sample along a line crossing one of two objects. The results were a little noisy because only $1 \times 10^{8}$ photon packets were used in the simulation. However, it can be seen that the reconstructed attenuation coefficient was very close to the true value of $1.02 \mathrm{~cm}^{-1}$.

Tissue scattering is considered the major obstacle in optical imaging because light loses its trajectory information owing to multiple scattering. To study the effect of tissue scattering on the tomographic system, we simulated a sample with a scattering coefficient higher than $30 \mathrm{~cm}^{-1}$. All the other parameters were the same as the previous simulation. Figure 4 shows an example of the projected attenuation acquired with and without a spatial filter. The locations corresponding to the two liquid bubbles had a smaller attenuation than expected. The measured attenuation peaked at positions corresponding to the inner surface of the vessel. This is because the longest path length in a cylindrical tissue is along the tangent line at the inner circle and can be derived as

$$
2\left[(r+t)^{2}-r^{2}\right]^{1 / 2}
$$

where $t$ is the vessel thickness and $r$ is the radius of the mandrel. For the example used in the simulation, $r=2 \mathrm{~mm}$ and $t=0.5 \mathrm{~mm}$, the longest path length for ballistic photons is $3.0 \mathrm{~mm}$. If the thickness is reduced to $250 \mu \mathrm{m}$, the longest path length will be $2.1 \mathrm{~mm}$. The required system dynamic range is proportional to the product of the path length and the attenuation coefficient. In the above example, if the attenuation coefficient is $50 \mathrm{~cm}^{-1}$, then the system dynamic range should be $\sim 90 \mathrm{~dB}$; if the attenuation coefficient increases to $100 \mathrm{~cm}^{-1}$, the required system dynamic range should be $\sim 180 \mathrm{~dB}$.

At positions closer to the inner circle, the attenuation measured with a spatial filter was higher than that measured without any angular discrimination. 


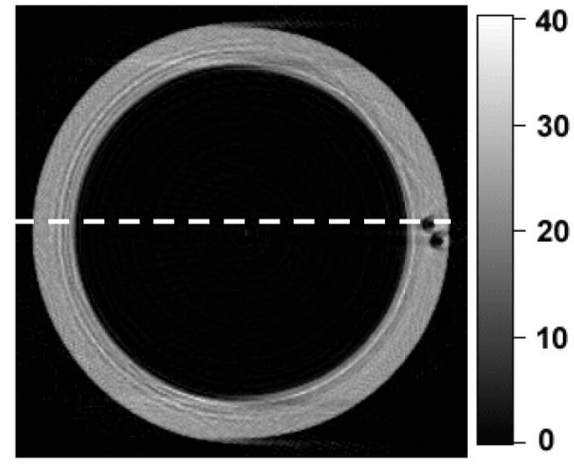

(a)



(b)

Fig. 5. Reconstruction of a tissue sample with a scattering coefficient of $30 \mathrm{~cm}^{-1}$. (a) Reconstructed image without a spatial filter. The gray-scale bar indicates the value of the reconstructed attenuation coefficient $\mu_{t}$ (in inverted centimeters). (b) Reconstructed attenuation coefficients along a line [the dashed line in (a)] crossing a liquid bubble.

Some of the photons measured at the corresponding positions were multiply scattered photons that had longer path lengths. Multiply scattered photons tend to have large angles and can be filtered out by a spatial filter. The relative weight of multiply scattered photons was higher at positions closer to the inner surface because the corresponding ballistic path length was longer and the number of nonscattered photons was smaller. Because of the multiple scattering effect, the actual attenuation coefficient at the inner surface of the tissue could be underestimated if multiply scattered photons were not fully rejected.

Figure 5 shows the reconstructed image. The two liquid bubbles are readily apparent. Figure 5(b) shows the attenuation coefficient curve along a line crossing one of the two objects. We compared the two curves that were obtained with and without a spatial filter. It can be seen that the attenuation coefficient $\mu_{t}$ was underestimated if no spatial filter was applied, especially at positions closer to the inner sample surface. Previous studies have indicated that the scattering coefficient of human aorta tissue is $\sim 40 \mathrm{~cm}^{-1}$ at the near-infrared region. ${ }^{16}$ The multiple scattering effects become severer as the scattering coefficient

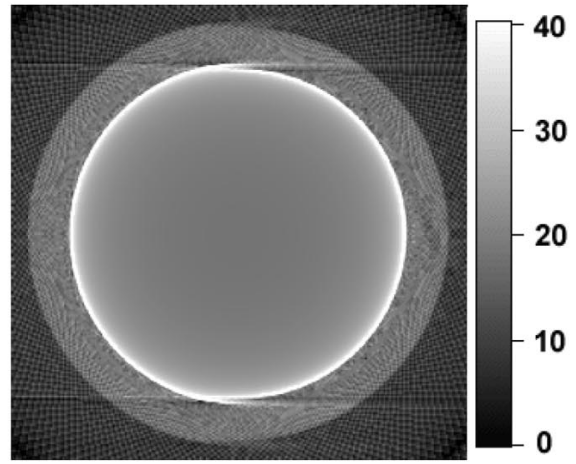

(a)



(b)

Fig. 6. Reconstructed image of a vessel on a solid mandrel. (a) The reconstructed image. The gray-scale bar indicates the value of the reconstructed attenuation coefficient $\mu_{t}$ (in inverted centimeters). (b) The reconstructed attenuation coefficient along a line crossing a liquid bubble.

gets larger. However, this effect also depends on the tissue thickness [Eq. (1)]. The vessel samples we are interested in usually have a thickness of a couple hundreds of microns. ${ }^{2}$ Therefore the scattering problems could be controlled by using spatial filtering. It is worth mentioning that once the multiple scattering becomes dominant, spatial filtering is no longer effective and the sample attenuation coefficient will be underestimated.

For practical vessel growing, the tissue is mounted on a solid mandrel, which is often made of stainless steel [Fig. 2(a)]. A simulation result is shown in Fig. 6 . The tissue sample had an absorption coefficient of $0.2 \mathrm{~cm}^{-1}$, a scattering coefficient of $20 \mathrm{~cm}^{-1}$, and a scattering anisotropic factor of 0.9 . The outer diameter of the tissue was $0.5 \mathrm{~cm}$, and the inner diameter of the tissue (or the mandrel diameter) was $0.4 \mathrm{~cm}$. The tissue was buried inside an index-matching fluid with a refractive index of 1.37 . It was assumed that the mandrel was coated with absorption material so that only $10 \%$ of the light would be reflected at the mandrel surface. A spatial filter with a passing angle of $0.1^{\circ}$ was used to remove multiply scattered photons. In Fig. 6, the two small objects are barely visible 


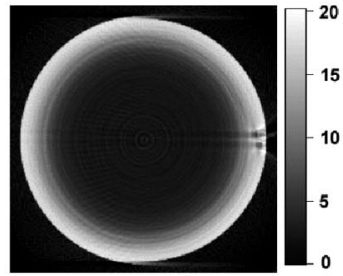

(a)

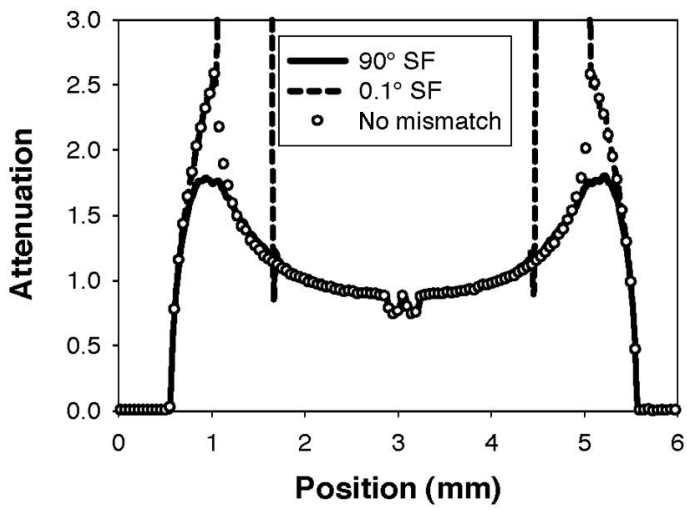

(c)



(d)

(b)

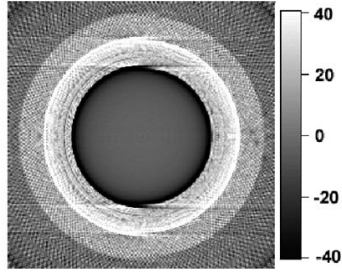



Fig. 7. Reconstructed image of a vessel on a transparent mandrel. (a) Reconstructed image without spatial filtering. The gray-scale bar indicates the value of the reconstructed attenuation coefficient $\mu_{t}$ (in inverted centimeters). (b) Reconstructed image obtained with a spatial filter that has an acceptance angle of $0.1^{\circ}$. (c) Projection data at $0^{\circ}$. (d) Ray-tracing map.

and have much less contrast than the objects in Fig. 5 . The tissue-mandrel boundary was overshot in the reconstructed image. Because the majority of the projections were blocked by the mandrel, they did not provide information on the vessel. Therefore using a solid mandrel is not an efficient method.

To overcome the problems caused by an opaque solid mandrel, we simulated cases in which a transparent mandrel [Fig. 2(b)] was used. Figure 7 demonstrates one of the examples. The tissue had a scattering coefficient of $20 \mathrm{~cm}^{-1}$, an absorption coefficient of $0.2 \mathrm{~cm}^{-1}$, and an anisotropy factor of 0.9 . The refractive index of the mandrel (1.40) is higher than that of the vessel (1.37). The mandrel had a thickness of only $50 \mu \mathrm{m}$. The whole setup was immersed in an index-matching medium with a refractive index of 1.37. The mandrel was filled with the same medium. Figure 7(a) shows the reconstructed image obtained without a spatial filter. Although the two objects were visible, there were some serious distortions near the tissue-mandrel interface. And close examination indicated that the reconstructed attenuation coefficients deviated significantly from the true values. When a spatial filter is used, the reconstructed images became even worse. The object contrast decreased, and there was a phantom ring starting from the tube-tissue junction toward the inside of the real tissue [Fig. 7(b)].

These observed effects were caused by the refractive-index mismatch between the tissue and the transparent mandrel. The projection data shown in Fig. 7(c) clearly illustrated these effects. From the projection data, it can be seen that the attenuation was smaller than the correct value (obtained without index mismatch) in the regions between the tissue outer boundary and the tissue inner boundary, whereas the attenuation was a little higher within the mandrel region. Figure 7(d) shows a simple raytracing diagram in which the scattering effect is not included. Also, the refractive index of the mandrel was increased to 1.47 for illustration purposes. Because of refraction at the tissue-mandrel interface, the photons entering the mandrel were diverged. Some photons were reflected at the inner surface of the mandrel because of total internal reflection. Therefore less light was recorded inside the mandrel, which led to a higher apparent attenuation within the mandrel and a lower attenuation outside the mandrel. The refractive-index mismatch problem also depended on the mandrel thickness. A thinner mandrel had less effect because the light deviation was minimized. However, the ultimate solution will be to use a mandrel material that has a refractive index that is matched with the vessel sample. Applying a spatial filter can produce additional distortions in the reconstructed images [Fig. 7(b)]. The photon deviation angle was bigger at locations closer to the tissue-mandrel boundary. These photons were rejected by the spatial filter, and a very high false attenuation was recorded [Fig. 7(c)], which appeared as a phantom ring in the reconstructed image.

Similar to the mandrel-vessel interface, the refractive-index mismatch between the surrounding culture media and the tissue also caused significant problems. Because the thickness of the sample was larger than that of the transparent mandrel tube, it produces an even severer distortion in the reconstructed images. An example is shown in Fig. 8, in which the refractive index of the surrounding medium was 1.365 , that of the vessel was 1.370 , and that of transparent mandrel was 1.370 (this is approxi- 


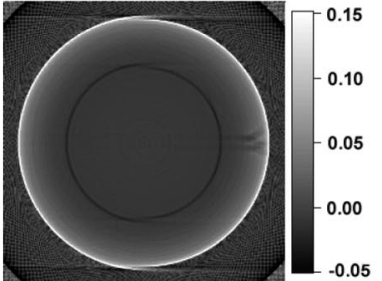

(a)

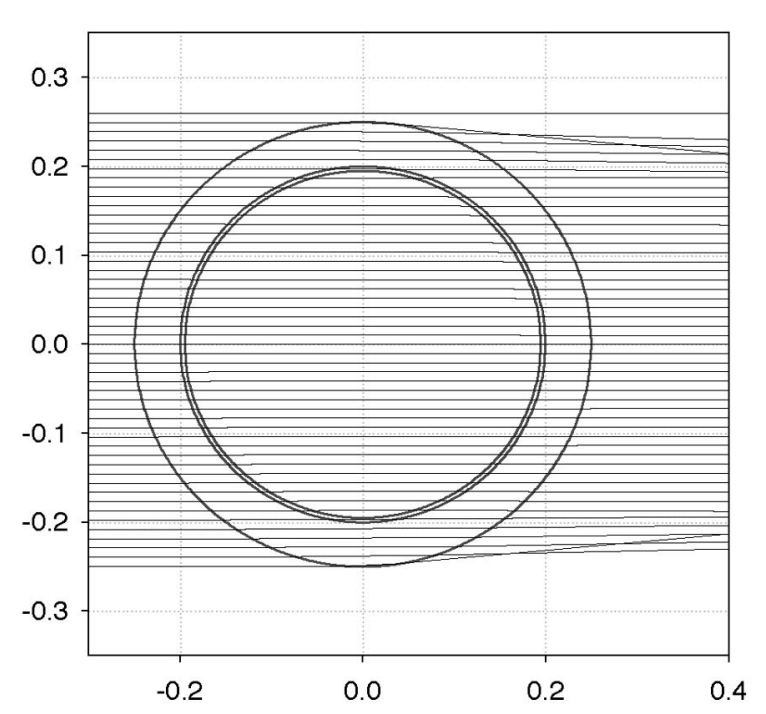

(c)

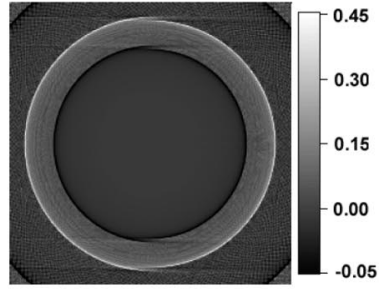

(b)
Fig. 8. Effect of background refractive-index mismatch. (a) Reconstructed image without spatial filtering. The gray-scale bar indicates the value of the reconstructed attenuation coefficient $\mu_{t}$ (in inverted centimeters). (b) Reconstructed image obtained with a spatial filter that has an acceptance angle of $0.1^{\circ}$. (c) Ray-tracing map.

mately the refractive index of $\mathrm{MgF}_{2}$ glass in the visible wavelength range). The tissue had an absorption coefficient of $0.2 \mathrm{~cm}^{-1}$, a scattering coefficient of $20 \mathrm{~cm}^{-1}$, and an anisotropy factor of 0.9 . In Fig. 8(a) it can be seen that the reconstructed image had an enlarged sample thickness; the contrast of the objects were also reduced. These distortions were caused by the light refraction at the tissue-medium interface. The light incident at the vessel boundary was bent toward the center of the vessel [Fig. 8(c)], which caused a decrease in attenuation within the inner vessel. At the same time, the projections from the vessel boundary decreased and led to the white edge distortion, which represented a very high attenuation coefficient. It was also noticed that if a spatial filter were used, the reconstructed image showed the correct vessel thickness [Fig. 8(b)] because most of the refracted light with high deflection angles was rejected. However, the reconstructed objects still had poor contrast. In addition, the reconstructed attenuation coefficients were incorrect.

The transmitted light was bent toward the center of the cylinder. The deviation angle $\Delta \alpha$ could be derived as

$$
\Delta \alpha=2\left[\sin ^{-1}\left(\frac{y}{r}\right)-\sin ^{-1}\left(\frac{n_{0}}{n_{t}} \frac{y}{r}\right)\right],
$$

where $n_{0}$ is the refractive index of the surrounding medium, $n_{t}$ is the refractive index of the vessel tissue, $r$ is the outer radius of the vessel, and $y$ is the distance between the incident light location and the center of the vessel (Fig. 2). The beam deviation is sensitive to the refractive-index mismatch. For the example shown in Fig. 8, the transmitted light has deviation angles greater than $1^{\circ}$ if the incident location $y$ $>0.926 r$, and the transmitted light has deviation angles greater than $0.1^{\circ}$ if the incident location $y$ $>0.233 r$. Therefore much of the transmitted light hit the wrong CCD pixels and produced an incorrect reconstructed image. If a spatial filter were used, those photons with large deviation angles would be rejected and would generate a false high-attenuation projection. In addition, the refracted light followed a different and longer path inside the sample, making it difficult to reconstruct the correct attenuation coefficients.

\section{Conclusion}

We have developed a Monte Carlo simulation program to simulate turbid media in a multilayer cylindrical structure. The program was applied to study transillumination laser tomography of engineered blood vessels. Different optical parameters can be assigned to the sample setup in the experiment. Our simulation studies indicated that the scattering problem can be controlled by use of simple angular discrimination that is due to the thin thickness of the vessel sample. However, the refractive-index mismatch produced significant distortions in the reconstructed images. For practical use of this method, the mandrel material and the surrounding culture medium should be chosen carefully so that their refractive indices match that of the vessel tissue. The simulation program could be helpful in the design of a practical optical transillumination system for vessel substitute imaging and be useful for the study of imaging artifacts.

\section{References}

1. R. M. Nerem and A. E. Ensley, "The tissue engineering of blood vessels and the heart," Am. J. Transplant. 4, 36-42 (2004).

2. N. L'Heureux, J. C. Stoclet, F. A. Auger, G. J. L. Lagaud, L. Germain, and R. Andriantsitohaina, "A human tissueengineered vascular media: a new model for pharmacological studies of contractile response," FASEB J. 15, 515-524 (2001).

3. R. H. Schmedlen, W. M. Elbjeirami, A. S. Gobin, and J. L. West, "Tissue engineered small-diameter vascular grafts," Clin. Plast. Surg. 30, 507-517 (2003).

4. R. R. Alfano and J. G. Fujimoto, eds., Advances in Optical Imaging and Photon Migration, Vol. 2 of Topics in Optics and Photonics Series (Optical Society of America, Washington, D.C., 1996).

5. B. Chance and R. R. Alfano, eds., Optical Tomography and Spectroscopy of Tissue: Theory, Instrumentation, Model, and Human Studies II, Proc. SPIE 2979, 1997. 
6. B. Das, K. Yoo, and R. R. Alfano, "Ultrafast time gated imaging," Opt. Lett. 18, 1092-1094 (1993).

7. S. Marengo, C. Pepin, T. Goulet, and D. Houde, "Time-gated transillumination of objects in highly scattering media using a subpicosecond optical amplifier," IEEE J. Sel. Top. Quantum Electron. 5, 895-901 (1999).

8. Y. Tetsuya, S. Tanosaki, Y. Sasaki, M. Takagi, A. Ishikawa, H. Taniguchi, B. Devaraj, and T. Akatsuka, "Fundamental imaging properties of transillumination laser computed tomography based on coherence detection imaging method," Anal. Sci. 18, 1329-1333 (2002).

9. J. C. Gladish, G. Yao, N. L'Heureux, and M. A. Haidekker, "Optical transillumination tomography for imaging of tissue-engineered blood vessels," Ann. Biomed. Eng. 33, 323-327 (2005).

10. D. Huang, E. A. Swanson, C. P. Lin, J. S. Schuman, W. G. Stinson, W. Chang, M. R. Hee, T. Flotte, K. Gregory, C. A. Puliafito, and J. G. Fujimoto, "Optical coherence tomography," Science 254, 178-1181 (1991).
11. D. Y. Churmakov, I. V. Meglinski, and D. A. Greenhalgh, "Amending of fluorescence sensor signal localization in human skin by matching of the refractive index," J. Biomed. Opt. 9, 339-346 (2004).

12. Y. Otani, T. Shimada, T. Yoshizawa, and N. Umeda, "Twodimensional birefringence measurement using the phase shifting technique," Opt. Eng. 33, 1604-1609 (1994).

13. L. Wang, S. L. Jacques, and L. Zheng, "MCML-Monte Carlo modeling of light transport in multi-layered tissues," Comput. Methods Programs Biomed. 47, 131-146 (1995).

14. L. Henyey and J. Greenstein, "Diffuse radiation in the galaxy," Astrophys. J. 93, 70-83 (1941).

15. L. A. Shepp and J. B. Kruskal, "Computerized tomography: the new medical x-ray technology,” Am. Math. Monthly 85, 420 439 (1978).

16. J. M. C. van Gemert, R. M. Verdaasdonk, E. G. Stassen, and G. Schets, "Optical properties of human blood vessel wall and plaque," Lasers Surg. Med. 5, 235-273 (1985). 